\title{
A ENFERMEIRA NO CUIDADO AOS PACIENTES SUBMETIDOS AO ECO-STRESS FARMACOLÓGICO: IMPLICAÇÕES PARA A PRÁTICA CLÍNICA
}

\author{
Marta Georgina Oliveira de GOES ${ }^{\mathrm{a}}$, Liana LAUTERT ${ }^{\mathrm{b}}$, Amália Fátima LUCENA
}

\section{RESUMO}

Estudo que teve por objetivos identificar os sinais e sintomas prévios e os ocorridos durante o exame de eco-stress farmacológico, descrever a atuação da enfermeira e os pressupostos para o cuidado de enfermagem na realização desse exame. Trata-se de pesquisa exploratório-descritiva, em uma unidade diagnóstica cardiológica de um hospital universitário de Porto Alegre. Foram estudadas, retrospectivamente, 246 fichas de registros de pacientes submetidos ao eco-stress, com quatro diferentes protocolos farmacológicos. A análise estatística apontou que os sinais e sintomas associados ao tipo de fármaco utilizado durante o exame foram, prevalentemente: angina típica, dor precordial, cansaço, cefaleia e extrassístoles. Esses resultados propiciaram conhecer melhor o eco-stress farmacológico, de forma a instrumentalizar a enfermeira para a prática clínica, tanto na organização da unidade para a realização do exame quanto no cuidado ao paciente submetido ao mesmo.

Descritores: Ecocardiografia sob estresse. Cuidados de enfermagem. Papel do profissional de enfermagem.

\section{RESUMEN}

Estudio que tuvo por objetivos, identificar los signos y síntomas previos, y los ocurridos durante el examen de ecoestrés farmacológico, y describir el rol de la enfermera y los fundamentos para los cuidados de enfermería durante la realización de este examen. Es un trabajo descriptivo, realizado en una unidad diagnóstica cardiológica de un hospital universitario de Porto Alegre, RS. Fueron estudiados, de forma retrospectiva, 246 expedientes médicos de pacientes sometidos al ecoestrés, con cuatro diferentes protocolos farmacológicos. El análisis estadístico demostró que los signos y sintomas fueron asociados al tipo de fármaco utilizado durante el examen, y estos fueron: angina típica, dolor precordial, cansancio, cefalea y extrasistoles. Estos resultados ayudaron a conocer mejor el ecoestrés farmacológico y preparar mejor a la enfermera para la práctica clínica, tanto en la organización del servicio donde se realizaron los exámenes como en los cuidados al paciente sometido al mismo.

Descriptores: Ecocardiografía de estrés. Cuidados de enfermería. Rol de la enfermera.

Título: El rol de la enfermera en la atención de pacientes sometidos al ecoestrés farmacológico: implicaciones en la práctica clínica.

\section{ABSTRACT}

The study aim was both to identify signs and symptoms previous to and during the pharmacological stress echocardiography test and to describe the nurse's role and nursing care principles for this exam. This is a descriptive study, carried out in cardiac care unit in a University Hospital in Porto Alegre, RS. Two hundred forty-six records of patients submitted to stress echocardiography were retrospectively reviewed, according to four different pharmacological schedules. The statistical comparison showed that signs and symptoms were related to the type of drug used during the exam, namely: typical angina, precordial ache, tiredness, headache and premature complexes. These results enabled a better understanding of pharmacological stress echocardiography and the instrumentalization of nurses in order to plan individualized and qualified nursing care assistance. Aside from helping develop nursing practices for the pharmacological stress echocardiography test, this knowledge could also be used by nurses who carry out their activities in institutions that use this diagnostic method.

Descriptors: Stress echocardiography. Nursing cares. Role of the nursing professional.

Title: Nursing care for patients undergoing pharmacological stress echocardiography: implications for clinical practice.

a Mestre em Enfermagem. Enfermeira da unidade de Hemodinâmica do Hospital de Clínicas de Porto Alegre, Porto Alegre, Rio Grande do Sul, Brasil.

b Doutora em Psicologia, Orientadora da dissertação de Mestrado. Diretora da Escola de Enfermagem da Universidade Federal do Rio Grande do Sul (UFRGS), Porto Alegre, Rio Grande do Sul, Brasil.

c Doutora em Ciências. Professora Adjunta da Escola de Enfermagem da UFRGS. Membro do Grupo de Estudo e Pesquisa em Enfermagem no Cuidado ao Adulto e Idoso, Porto Alegre, Rio Grande do Sul, Brasil. 


\section{INTRODUÇÃO}

A atuação de enfermeiras em serviços diagnósticos ainda é pequena em nosso meio. Entretanto, com o avanço da tecnologia este espaço tem sido ampliado à medida que aumenta o número e a complexidade dos exames, o que leva à necessidade do cuidado de enfermagem especializado aos indivíduos submetidos a tais procedimentos.

O eco-stress farmacológico ${ }^{(1)}$ é uma técnica ecocardiográfica reconhecida como eficaz para avaliação dos pacientes com diagnóstico ou suspeita de doença arterial coronariana. Por meio dele é possível quantificar a isquemia e/ou a viabilidade de segmentos do miocárdio, baseado na visualização das alterações segmentares de contração do músculo cardíaco, quando submetido a uma condição de stress induzido por fármacos, em comparação com a contração em repouso ${ }^{(2)}$.

As drogas mais utilizadas para produzir este stress farmacológico ${ }^{(3,4)}$ são a dobutamina, o dipiridamol e a atropina. A dobutamina é uma catecolamina sintética que age nos receptores $\beta$ - 1 adrenégicos do miocárdio, produzindo um aumento na frequência cardíaca $(\mathrm{FC})$, na condução atrioventricular e na contratilidade cardíaca. O dipiridamol ${ }^{(2,5)}$ é um vasodilatador que reduz o suprimento de oxigênio ao miocárdio, através da redistribuição do fluxo sangüíneo pela estimulação dos receptores $\mathrm{A}_{2} \mathrm{a}$ adenossinérgicos, presentes no endotélio e nas células musculares lisas das arteríolas coronárias. A atropina é um agente antimuscarínico que aumenta a FC e contribui para o incremento da sensibilidade $^{(4)}$ do teste ao ser associada a dobutamina e o dipiridamol. Estas drogas podem ser combinadas conforme protocolos já estabelecidos.

As referências encontradas na literatura americana sobre a assistência de enfermagem na realização do eco-stress salientam a ampliação do espaço de atuação da enfermeira no laboratório de ecocardiografia ${ }^{(6)}$ e descrevem a experiência das registered nurse sonographers ${ }^{(7)}$, as quais realizam o teste com dobutamina, sob supervisão de um médico. Em pesquisa, realizada com dois grupos, um com a participação da enfermeira e outro sem, foi considerada segura a realização do teste pela enfermeira $^{(7)}$. Outros autores também descrevem que a participação das enfermeiras em todas as etapas do teste confere segurança e efetividade ${ }^{(8,9)}$ e, de acordo com o idealizador deste exame, a sua atuação é aconselhada antes do procedimento e até o final do mesmo e portanto, propõe ações para sua performance ${ }^{(10,11)}$.

Nesta mesma perspectiva, ao se iniciar a realização desse exame em uma unidade de exames cardiológicos não invasivos de um hospital universitário do sul do Brasil, foi incluída a participação de uma enfermeira. Esta foi orientada pelo médico responsável e buscou informações, por meio de leituras e discussões clínicas, para sua atuação.

Dessa experiência, emergiu o desafio de estudar essa prática considerando-se que é um campo com produção cientifica limitada na área da enfermagem até o momento.

Assim, realizou-se um estudo(1) que teve por objetivos identificar os sinais e sintomas prévios e os ocorridos durante o exame de pacientes submetidos ao eco-stress, associados aos protocolos farmacológicos utilizados e, descrever a atuação da enfermeira e os pressupostos para o cuidado de enfermagem na realização deste exame.

\section{METODOLOGIA}

Este artigo é o recorte de um estudo exploratório-descritivo, realizado em uma unidade diagnóstica cardiológica não invasiva de um hospital universitário de Porto Alegre ${ }^{(1)}$.

A amostra constou de 246 fichas de registros efetuados pela enfermeira durante o atendimento a pacientes submetidos ao eco-stress. Neste instrumento constavam as anotações referentes a sinais e sintomas prévios ao exame e os apresentados durante a realização do procedimento, além do protocolo utilizado.

Os dados foram analisados de forma estatística, utilizando-se medidas de tendência central. $\mathrm{O}$ teste $t$ pareado foi usado para testar a diferença entre as médias dos valores sistólicos e diastólicos da pressão arterial (PA) e FC, o teste de qui-quadrado $\left(\chi^{2}\right)$ de Pearson para testar as diferenças entre os grupos formados pelos protocolos e, o de McNemar para testar a diferença nas amostras combinadas (sinais e sintomas antes e após o exame). Para comparar as variações da FC antes, na fase de viabilidade e no pico do stress, assim como da pressão sistólica e diastólica foi empregado o teste de Kruskal-Wallis one way ANOVA (análise de variância). O teste de Dunn foi usado para avaliar a significância destas alterações e o nível de significância adotado foi de $\mathrm{p}<0,05$.

O estudo foi aprovado pelo Comitê de Ética em Pesquisa da instituição $\left(n^{\circ}\right.$ 99-317) e foi assinado 
pelas pesquisadoras um termo de responsabilidade para o uso dos dados.

\section{RESULTADOS E DISCUSSÃO}

Analisaram-se dados contidos nas fichas de registro de 246 pacientes, destes 103 (41,9\%) do sexo feminino e $143(58,1 \%)$ do sexo masculino. A faixa etária predominante, em ambos os sexos, foi a de 60 a 69 anos, com 77 (31,3\%) pacientes. Contudo, não houve diferenças entre os grupos das faixas etárias com o uso do coeficiente de correlação de Pearson $(\mathrm{p}=0,64)$.

As condições físicas e clínicas dos pacientes, bem como os propósitos da realização do exame, determinaram a escolha entre um dos quatro protocolos: dipiridamol, dobutamina, dipiridamol ultra fast (UF) e dipiridamol com dobutamina. O maior número de pacientes $131(53,2 \%)$ foi submetido ao protocolo dipiridamol $U F$, seguido do com dobutamina 89 (36,1\%), com dipiridamol $18(7,3 \%)$ e dipiridamol e dobutamina oito pacientes (3,2\%).

A angina típica foi o sintoma prévio ao exame mais frequente $122(49,5 \%)$, seguido pela queixa de dor precordial 31 (12,9\%).

Como o eco-stress é indicado para pacientes com suspeita de cardiopatia isquêmica ${ }^{(2,4)}$, cujo sintoma mais frequentemente é a dor anginosa, era esperado que este sintoma fosse o mais relatado pelos pacientes.
A dor precordial ou torácica, embora leve, pode ser de origem cardíaca, sendo motivo para muitos pacientes buscarem auxílio médico. Entretanto, muitas vezes, pode estar associada a outras causas tais como a esofagite de refluxo, hérnia de hiato, espasmo esofagiano, ansiedade, doença de origem pulmonar, pericardite, compressões de raízes nervosas, doença de natureza musculoesquelética, ou ainda, relacionada à disfunção de órgãos abdominais $^{(12,13)}$. Além disto, existem outras condições em que a dor torácica pode estar presente, ser de origem cardíaca, mas sem lesão coronária: angina vasoespástica ${ }^{(14)}$ ou microvascular (denominada síndrome $\mathrm{X}$ ), prolapso da valva mitral e miocardites agudas. Nas condições provocadas pela alteração na relação entre a oferta e a demanda de oxigênio pelo miocárdio se incluem, ainda a estenose valvar aórtica, cardiomiopatia hipertrófica, intoxicação por cocaína ${ }^{(15)}$, miocardiopatia dilatada e taquiarritmias ${ }^{(1)}$.

Esta diversidade de etiologias da dor torácica precisa ser cuidadosamente investigada e avaliada pela enfermeira. Para isto, ela deve realizar anamnese e exame físico do paciente, pois é necessário associar uma queixa a outros sinais e sintomas para construir um diagnóstico acurado e embasar o planejamento da assistência.

Neste estudo foram observados sete sinais e sintomas na realização do exame de eco-stress (Tabela 1). Nos pacientes que fizeram o teste com dipiridamol

Tabela 1 - Distribuição dos sinais e sintomas evidenciados nos pacientes durante o eco-stress farmacológico em três protocolos farmacológicos utilizados. Porto Alegre, RS, 1999.

\begin{tabular}{lcccccc}
\hline & \multicolumn{6}{c}{ Protocolos } \\
\cline { 2 - 7 } Sinais e Sintomas & \multicolumn{2}{c}{$\begin{array}{c}\text { Dipiridamol } \\
(\mathrm{n}=18)\end{array}$} & \multicolumn{2}{c}{$\begin{array}{c}\text { Dobutamina } \\
(\mathrm{n}=89)\end{array}$} & $\begin{array}{c}\text { Dipiridamol ultra fast } \\
(\mathrm{n}=131)\end{array}$ \\
\cline { 2 - 7 } & $\mathrm{F}$ & $\%$ & $\mathrm{~F}$ & $\%$ & $\mathrm{~F}$ & $\%$ \\
\hline Cefaléia & 03 & $16,7 \%$ & 02 & $2,6 \%$ & 29 & $17,6 \%$ \\
Angina típica & $\mathrm{O}$ & $22,2 \%$ & $\mathrm{O}$ & $7,8 \%$ & 12 & $9,6 \%$ \\
Dor precordial & $\mathrm{O}$ & $16,7 \%$ & $\mathrm{O} 1$ & $1,3 \%$ & 11 & $8,7 \%$ \\
Cansaço & $\mathrm{O}$ & $11,1 \%$ & $\mathrm{O} 1$ & $1,3 \%$ & 09 & $7,1 \%$ \\
Extrassístoles ventriculares & - & - & 13 & $15 \%$ & 01 & $0,8 \%$ \\
Tonturas & - & - & - & - & 04 & $3,2 \%$ \\
Taquicardia ventricular & - & - & 02 & 2,6 & - & - \\
Total & 12 & $66,7 \%$ & 24 & $32,3 \%$ & 66 & $47 \%$ \\
\hline
\end{tabular}


$U F$, tanto no período que antecedeu o exame como após o mesmo, obteve-se diferença estatisticamente significativa $(\mathrm{p}=0,00)$ para os sinais e sintomas de angina típica e cefaléia $(\mathrm{p}=0,02)$, dor precordial $(\mathrm{p}=0,01)$ e cansaço $(\mathrm{p}=0,02)$. O mesmo ocorreu nos pacientes submetidos ao protocolo com dobutamina para angina típica e extrassístoles ventriculares (EV) $(\mathrm{p}=\mathrm{0}, \mathrm{03})$. Nos oito pacientes submetidos ao protocolo com dipiridamol-dobutamina não se observou sinais ou sintomas durante a realização do exame.

Alguns protocolos podem desencadear maior sintomatologia como é o caso do dipiridamol ${ }^{(1,4,5)}$, o qual pode ocasionar graus variados de cefaléia, calor e rubor facial, cansaço, ardência no sítio de punção venosa e a possibilidade de dor precordial e/ou angina típica. Estes dados foram corroborados por este estudo, principalmente, no uso do dipiridamol $U F$, quando comparado com os outros dois testes estudados. Não se identificou o calor e rubor facial e a ardência no sítio de punção.

A dobutamina pode ocasionar palpitações, dispnéia, sensação de arrepio $^{(16)}$ no couro cabeludo e corpo, tremores e $\mathrm{EV}^{(3,4)}$. Neste estudo se identificou a presença de cefaléia, angina típica, dor precordial, cansaço, extrassístoles e taquicardia ventricular. Os sintomas de tremores e sensação de arrepio no couro cabeludo não foram identificados.

A ocorrência de cefaléia ${ }^{(5)}$, no uso do dipiridamol, é atribuída a sua ação vasodilatadora. Todavia, conforme o idealizador do eco-stress ${ }^{(10,11)}$, somente em $2 \%$ dos casos, a queixa de cefaléia impede a continuação do exame. Neste estudo, nenhum exame foi interrompido, pois os pacientes toleraram bem o sintoma, mesmo sendo alto o percentual de relatos se considerarmos que não o referiram antes do exame.

O elevado percentual de pacientes que apresentou angina típica $(39,6 \%)$ pode ser justificado pelo desfecho do teste, o qual foi positivo para a isquemia miocárdica em 22 (28,9\%) dos pacientes submetidos ao protocolo de dobutamina; e em 33 $(26,4 \%)$ daqueles que realizaram o teste com dipiridamol $U F$.

A ocorrência de dor precordial, principalmente, no grupo que foi submetido ao exame com os protocolos dipiridamol e dipiridamol $U F$ também pode ser atribuída às alterações hemodinâmicas, decorrentes do efeito farmacológico, as quais provocam um aporte insuficiente de oxigênio ao miocárdio sem isquemia coronariana ${ }^{(5,10,11)}$.

A queixa de tontura foi referida somente pelos indivíduos submetidos ao teste com dipiridamol
UF e pode estar associado ao incremento da dose, além do potencial efeito vasodilatador do fármaco. O cansaço pode ser atribuído à "simulação do exercício”, uma vez que o dipiridamol ${ }^{(2,5)}$ ao estimular os receptores da adenosina reduz a perfusão nas camadas subendocárdicas, desencadeando um efeito semelhante ao provocado pelo exercício.

As extrassístoles podem ter sido provocadas e/ou acentuadas pelo uso da dobutamina, droga com potencial arritmogênico, em pacientes que as apresentavam previamente. Neste estudo, 5,2\% dos pacientes submetidos ao teste com este protocolo que apresentavam esta alteração antes do exame, a apresentaram no seu decorrer. Estudos apontam que as EV podem ser a causa da interrupção do exame em cerca de $10 \%$ das vezes ${ }^{(2,3)}$. As extrassístoles no uso do protocolo com dipiridamol $U F$ podem estar associadas ao esforço induzido pelo exame.

As variações percentuais da PA sistólica, antes e durante o eco-stress, apresentaram correlações significativas pelo teste de Dunn para comparações múltiplas $(\mathrm{p}<0,05$ equivale a $\mathrm{Q}=2,639)$, nos grupos dos pacientes submetidos ao teste com dobutamina e dipiridamol $U F(Q=4,70)$ e no grupo dobutamina e dipiridamol $(Q=4,22)$, o que sugere variação maior no grupo que utilizou a dobutamina. O aumento da PA sistólica ${ }^{(3-4)}$ é relatado como um paraefeito do fármaco.

As variações PA diastólica não apresentaram diferenças significativas ( $Q$ entre 0,26 e 2,56) no cruzamento dos protocolos. As médias da FC, durante a realização do exame, aumentaram nos quatro protocolos. Este sinal é descrito como um efeito da atropina e dobutamina, mas também pode ser decorrente da ansiedade e da dor.

A ocorrência de sinais ou sintomas durante a realização do exame foi relativamente baixa, o que pode estar associado ao acompanhamento dos profissionais. Salienta-se a participação da enfermeira que possui papel importante no monitoramento dos sinais e sintomas apresentados pelo paciente durante a aplicação do protocolo.

\section{Implicações para a prática clínica e atribuições da enfermeira na unidade de cardiologia diagnóstica não invasiva}

Os resultados desta pesquisa, aliados à experiência prática da enfermagem, na unidade de cardiologia diagnóstica não invasiva, favoreceu a construção de uma base de conhecimentos para a 
sustentação dos pressupostos do cuidado de enfermagem na realização do eco-stress com acurado direcionamento das ações e consequentemente melhores resultados.

As funções da enfermeira na realização do eco-stress iniciam no dia que antecede o exame, quando toma conhecimento do número de pacientes agendados. Além de um estoque mínimo de medicações, conforme o protocolo a ser utilizado, será necessário um desfibrilador cardíaco com material para reanimação cardiorrespiratória, medicações-padrão para uso em ressuscitação cardiopulmonar, eletrocardiógrafo de doze derivações, bomba de infusão e cronômetro.

No dia do exame, a enfermeira deve coletar os dados do paciente e orientá-lo sobre a realização do procedimento, considerando o protocolo, o interesse e as dúvidas do paciente relacionadas ao exame ${ }^{(1)}$.

Conforme verificado nos resultados deste estudo e nos relatos da literatura, os pacientes que realizarem o teste com dobutamina devem ser preparados para a possibilidade de apresentarem sensação de arrepio no couro cabeludo, palpitações e angina típica e os indivíduos submetidos ao protocolo com dipiridamol que podem sentir cefaléia, angina típica, dor precordial, cansaço e ardência no sítio de punção. Para ambos os grupos que também utilizarem a atropina é esperado visão borrada, sensação de boca seca e palpitações ${ }^{(1,4)}$.

Sabe-se, que o conteúdo das orientações fornecidas ao paciente pela enfermeira é importante, o que requer uma boa relação interpessoal para estabelecer a confiança ao se submeter a um exame relacionado ao coração, o qual possui um significado simbólico.

Em razão disso, a enfermeira deve utilizar todos os recursos para que a interação estabelecida com o paciente seja clara e efetiva. Assim, antes e durante a realização do exame ela deve estar atenta para a comunicação não-verbal e para-verbal e utilizar do toque instrumental e expressivo ${ }^{(17)}$, que podem favorecer a sua aproximação.

A enfermeira necessita coletar dados para anamnese a qual deve conter informações sobre as doenças prévias e as que impedem a utilização de dipiridamol (asma, bronquite, acidente vascular encefálico, há menos de três meses), da atropina (glaucoma, prostatismo), da dobutamina (hipertensão arterial grave, história de arritmias, hipertensão pulmonar) e as associadas com a cardiopatia ${ }^{(18)}$, como a hipertensão arterial e diabete melito. O histórico de alergias é importante, pois visa o efetivo atendimento em situação de urgência. Além disso, os motivos que levaram a realização do exame, bem como os sinais e sintomas prévios a ele, a fim de compará-los com possíveis manifestações durante o procedimento.

A informação sobre o uso de medicações é essencial, especialmente quanto aos beta-bloqueadores, inclusive os de uso tópico presentes nos colírios $^{(19)}$ indicados para o tratamento do glaucoma, pois podem interferir no resultado do exame devido ao seu efeito bradicárdico. No exame físico se deverá atentar para a PA e a FC, que serão monitoradas durante todo o exame.

Para uma boa anamnese e exame físico é necessário desenvolver um instrumento para os registros com uma apresentação gráfica que facilite o preenchimento durante a realização do eco-stress. Após o exame, a enfermeira realiza a evolução no prontuário do paciente sobre as condições de realização do mesmo, as intercorrências e as condutas adotadas.

As informações registradas, associadas ao conhecimento técnico e científico da enfermeira sobre o eco-stress propiciam subsídios para avaliar o paciente, distinguir entre os sinais e sintomas, decorrentes do fármaco utilizado, daqueles anteriormente apresentados e assim prestar o cuidado individualizado e diminuir as complicações decorrentes do exame.

Durante a realização do exame, cabe ao médico verificar a qualidade da janela acústica para obtenção das imagens cardíacas no ecocardiógrafo e, somente então, a enfermeira punciona um vaso periférico e instala solução fisiológica para sua manutenção. $\mathrm{O}$ acesso venoso para realização deve ser obtido por meio da inserção de um cateter intravascular periférico flexível, com calibre compatível ao vaso escolhido, preferencialmente, no membro superior direito. Caso não seja possível, deve-se puncionar o membro superior esquerdo, evitando-se punções próximas e/ou na fossa cubital, pois dificultam a verificação da PA que deverá ser monitorada com frequência.

Com base no peso do paciente a enfermeira prepara as medicações necessárias deixando-as dispostas pela ordem do uso. As medicações antagonistas devem ficar à disposição, mas o seu preparo pode ser efetuado no momento da indicação. Ao infundir os fármacos deve-se determinar com precisão o tempo de infusão da droga. 
O exame deve ser cronometrado para que o teste transcorra dentro do tempo previsto, descontando-se o tempo para ajustar o volume $(\mathrm{ml} / \mathrm{h})$ na bomba de infusão, a fim de que todas as etapas tenham a mesma duração. O dipiridamol, por causa de sua formulação, pode provocar sensação de ardência no local da punção, o qual pode ser minorado aumentando a diluição da droga e o gotejo da solução fisiológica concomitante. A PA e FC são verificadas e calculado(1) o valor da FC máxima e submáxima. Deste modo, os profissionais têm conhecimento do limite que o paciente pode atingir com segurança.

Outro aspecto importante a ser considerado pela enfermeira é o conforto do paciente na mesa de exame. A posição comumente utilizada para realização do eco-stress é o decúbito lateral esquerdo, contudo, para a obtenção de imagens de boa qualidade, às vezes, é necessário que o paciente mantenha o tronco em leve torção para o lado direito. Para que esta posição se torne tolerável é preciso colocar um coxim de apoio na região dorsal, abaixo das escápulas, para evitar a dor muscular decorrente do posicionamento e que pode gerar confusão no diagnóstico diferencial entre a dor muscular e a de natureza isquêmica atípica.

Após a obtenção das imagens cardíacas basais pelo médico, a enfermeira inicia a infusão das drogas, manualmente ou com bomba de infusão, sendo que a partir de então o exame passa a ser cronometrado. Atualmente, foi acrescido o esforço isométrico ao protocolo com dobutamina, no qual o paciente realiza compressão manual de uma bola ou movimentos com os membros inferiores que simulam o pedalar até atingir a $\mathrm{FC}$ submáxima. O esforço aumenta o consumo de oxigênio miocárdico por elevar a resistência vascular periférica e o nível plasmático de noradrenalina ${ }^{(20)}$, induz mais rapidamente a elevação da FC e possibilita a utilização de doses menores de dobutamina e dispensar o uso de atropina.

A monitorização eletrocardiográfica é contínua, tanto em doze derivações no eletrocardiógrafo, como em três derivações no ecocardiógrafo. Durante o exame, a enfermeira permanece atenta ao paciente, observando-o e buscando antecipar-se às suas alterações.

A verificação e o registro dos valores de PA e FC são realizados a intervalos entre três e quatro minutos, preconizados pelos protocolos, para o ajuste das doses e para incrementar o trabalho cardíaco, evidenciado pelo aumento nos valores da PA e FC ou pelo sofrimento miocárdico, demonstrado pelas alterações eletrocardiográficas e angina típica.

Os critérios para interrupção ou conclusão do exame $^{(2,4,10,11)}$ são os sinais indicativos de isquemia miocárdica (angina típica e desnivelamento do segmento ST no eletrocardiograma), atingir a FC máxima, ter recebido a dose máxima de medicação ou a confirmação ecocardiográfica de alteração do segmento cardíaco. $\mathrm{O}$ teste também pode ser interrompido pela presença de desconforto intolerável, hipertensão arterial (pressão sistólica > $220 \mathrm{mmHg}$ e diastólica > $120 \mathrm{mmHg}$ ), hipotensão arterial (diminuição da PA sistólica em $30 \mathrm{mmHg}$ ), arritmias supraventriculares (taquicardia supraventricular e fibrilação ventricular) e ventriculares (taquicardia ventricular, extrassístoles ventriculares freqüentes e polimórficas).

A supressão desses sintomas pode ser feita pela administração do antídoto. A medicação antagonista ${ }^{(4,5)}$ da dobutamina é o metoprolol e do dipiridamol, a aminofilina. Ao término do exame ${ }^{(1)}$ que transcorreu de maneira normal, será realizado um eletrocardiograma de doze derivações e verificada a PA, para a certificação de que as condições do paciente ao final sejam similares ao período que antecedeu o exame. Os eletrodos e o acesso venoso periférico são removidos. O paciente é então, auxiliado a sentar-se vagarosamente na mesa de exame sendo, posteriormente, encaminhado para trocar a roupa no vestiário.

Os pacientes que apresentarem intercorrências serão acompanhados pela equipe até a estabilização do quadro. Aqueles que não as apresentaram podem ser liberados após trinta minutos.

Na finalização do exame, ressalta-se a necessidade do registro no prontuário do paciente sobre o tipo de protocolo utilizado, a presença ou não de intercorrências e os sinais vitais do paciente antes e ao término do exame, bem como as condutas adotadas.

\section{CONSIDERAÇÕES FINAIS}

Os sinais e sintomas apresentados pelos pacientes durante o eco-stress contribuíram para ampliar o conhecimento e qualificar a prática da enfermeira na unidade de cardiologia, principalmente, nos cuidados que antecedem o exame, como o preparo da unidade e orientação do paciente, o que proporciona maior segurança a todos. Acredita-se ainda, que estes achados também poderão ser 
úteis para profissionais de diversas instituições que aplicam ou que iniciarão a utilizar este método diagnóstico.

A evolução tecnológica na área diagnóstica apresentou grandes avanços nas últimas décadas e o eco-stress representa um deles, sendo aceito como uma possibilidade concreta para o diagnóstico da isquemia miocárdica, com custo relativamente baixo e de rápida interpretação. Todavia, necessita de condições específicas à sua realização, principalmente no que diz respeito à qualificação da equipe de profissionais, com vistas à segurança do paciente.

Como limitante neste estudo, cita-se a dificuldade em comparar os seus achados com a literatura de enfermagem, uma vez que são poucos os estudos desenvolvidos, possivelmente pelo reduzido número de enfermeiras atuantes nesta área. Embora, exista literatura que descreva os benefícios da participação da enfermeira nos cuidados ao paciente que realiza o exame, esta ainda é escassa. Frente a isso, entende-se que pesquisas como estas podem ampliar o corpo de conhecimentos da profissão e qualificar o cuidado prestado aos pacientes na área da cardiologia diagnóstica.

\section{REFERÊNCIAS}

1 Goes MGO. A enfermeira no cuidado aos pacientes submetidos ao eco-stress farmacológico [dissertação]. Porto Alegre: Universidade Federal do Rio Grande do Sul; 2000.

2 Picano E, Molinaro S, Pasanisi E. The diagnostic accuracy of pharmacological stress echocardiography for the assessment of coronary artery disease: a meta-analysis. Cardiovasc Ultrasound. 2008; 6:30.

3 Minardi G, Manzara C, Pulignano G, Pino PG, Pavaci $H$, Sordi M. Feasibility, safety and tolerability of accelerated dobutamine stress echocardiography. Cardiovasc Ultrasound. 2007; 5:40.

4 Pellikka P, Nagueh SF, Elhendy AA, Kuehl CA, Sawada, SG. American society of echocardiography recommendations for performance, interpretation, and application of stress echocardiography. J Am Soc Echocardiogr. 2007; 20(9):1021-1041.

5 Rossi A, Moccetti T, Faletra F, Cattaneo P, Rossi M, Pasotti E et al. Dipyridamole stress echocardiography stratifies outcomes of asymptomatic patients with recent myocardial revascularization. Int J Cardiovasc Imaging. 2008; 24:495-502.
6 Laurienzo MD. Transesophageal dobutamine stress echocardiography: the nurse's role. J Cardiovasc Nurs. 1995; 9(4): 24-35.

7 Bremer ML, Monahan KH, Stussy VL, Miller FA, Seward JB, Pellikka PA. Safety of dobutamine stress echocardiography supervised by registered nurse sonographers. J Am Soc Echocardiogr. 1998; 11(6): 601-605.

8 Kane G, Hepinstall MJ, Kidd GM, Kuehl CA, Murphy AT, Nelson JM, et al. Safety of stress echocardiography supervised by registered nurses: results of a 2-year audit of 15,404 patients. J Am Soc Echocardiogr. 2008; 21(4): 337-416

9 Gilman G, Nelson JM, Murphy AT, Kidd GM, Stussy VL, Kyle W, et al. The role of the nurse in clinical echocardiography. J Am Soc Echocardogr. 2005; 31 : 773-7.

10 Picano E. Stress Echocardiography. $3^{\text {th }}$ ed. Berlim: Springer; 1997.

11 Picano E. Ecocardiografia de Estresse - Apêndice: Ecocardiografia de Estresse no Brasil e em Portugal. 3a ed. Rio de Janeiro: Revinter; 2000.

12 Lenfant C. Chest pain of cardiac and noncardiac origin. Metabolism. 2010; 59 (Suppl 1): 41-6.

13 Winters ME, Katzen SM. Identifying chest pain emergencies in the primary care setting. Prim Care. 2006; 33(3):625-42.

14 Bogaz FA, Saroute ANR, Tsutsui JM, Kowatsch I, Neto FMO; Nicolau JC, et al. Vasoespasmo coronariano induzido pela ecocardiografia sob estresse pela dobutamina-atropina. Arq Bras Cardiol. 2006; 87(6): 250-253.

15 Lippi G, Plebani M, Cervellin G. Cocaine in acute myocardial infarction Adv Clin Chem. 2010; 51:53-70.

16 Vidal I. Piloereção: um efeito colateral da administração intravenosa de dobutamina Arq Bras Cardiol. 2009; 92(4): 290-293

17 Lucena AF, Goes MGO. O processo de comunicação no cuidado do paciente submetido ao eco-stress: algumas reflexões. Rev Gaúcha Enferm. 1999; 20(n esp): 37-48.

18 Feijó MKEF, Lutkmeier R, Ávila CW, Rabelo ER. Fatores de risco para doença arterial coronariana em pacientes admitidos em unidade de hemodinâmica. Rev Gaúcha Enferm. 2009; 30(4):641-7. 
19 Cordovil A, Matos FQ, Campos Filho O, Paranhos Jr. A, Rodrigues ACT, Albernaz MV, et al. Betabloqueador tópico pode determinar resultados inconclusivos no ecocardiograma sob estresse com dobutamina em pacientes com glaucoma. Arq Bras Cardiol. 2007; 89(1): 60-63.
20 Barretto RBM, Gimenes VM. Dopplerecocardiografia na avaliação de pacientes com angina estável [Internet]. 2003 [citado 2010 nov 22];3(2):204213. Disponível em http://www.socesp.org.br/ revistasocesp/edicoes/volume13/v13_no2_tx03. asp? posicao $=$ completo $\& \mathrm{v}=\& \mathrm{n}=$.

Endereço do autor / Dirección del autor / Author's address

Marta Georgina Oliveira de Góes

Avenida Jerônimo de Ornelas, 688, ap.602, Santana 90040-340, Porto Alegre, RS

E-mail:mgogoes@gmail.com 